\title{
Eficiência do melaço como fonte de carbono na remoção de nitrito em lixiviados de aterros sanitários
}

\author{
Molasses efficiency as carbon source in nitrite removal from landfill leachates
}

Luana Zilz', Joel Dias da Silva², Adilson Pinheiro

$\square$

\section{RESUMO}

Neste trabalho simulou-se a desnitrificação via nitrito em lixiviado de aterro sanitário, operando-se em escala laboratorial através de um reator em batelada sequencial (RBS) adicionando-se melaço de cana como fonte externa alternativa de carbono. As razões $\mathrm{DBO}_{\text {lixiviado+melacco }} / \mathrm{N}$ $\mathrm{NO}_{\mathrm{x}}$ estudadas foram 0,37; 4; 12 e 14 com tempo de reação anóxica de 4,33 h (DBO/N-NO $=0,37 ; 4$ e 14) e $22 \mathrm{~h}\left(\mathrm{DBO} / \mathrm{N}-\mathrm{NO}_{\mathrm{x}}=12\right)$. A taxa específica de desnitritação foi de 0,07 kg nitrito. $\mathrm{kg}^{-1}$ sólidos suspensos voláteis (SSV)*dia após 4,33 h de reação anóxica nos ensaios sem adição de melaço de cana e 0,19 e 0,12 kg nitrito. $\mathrm{kg}^{-1} \mathrm{SSV}^{*}$ dia nos ensaios realizados com adição de melaço de cana, fornecendo uma razão $\mathrm{DBO} / \mathrm{N}-\mathrm{NO}_{x}$ de 4 e 14, respectivamente. Ampliando-se o tempo de reação anóxica para 22 h e realizando os ensaios sob razão $\mathrm{DBO} / \mathrm{N}-\mathrm{NO}_{x}$ de 12, a taxa específica de desnitritação foi aumentada para 0,55 kg nitrito. $\mathrm{kg}^{-1}$ SSV*dia.

Palavras-chave: lixiviado; nitrito; desnitrificação; melaço de cana; reator em batelada sequencial.

\begin{abstract}
In this work, the denitrification was simulated via nitrite at landfill leachate, operating at laboratory scale through a sequencing batch reactor (SBR) adding cane molasses as an alternative external source of carbon. The reasons $\mathrm{BOD}_{\text {landfill leachate+molasses }} / \mathrm{N}-\mathrm{NO}_{x}$ studied were 0.37 , 4, 12 and 14 with anoxic reaction time of $4.33 \mathrm{~h}\left(\mathrm{BOD} / \mathrm{N}-\mathrm{NO}_{\mathrm{x}}=\mathrm{O} .37,4\right.$ and 14$)$ and $22 \mathrm{~h}\left(\mathrm{BOD} / \mathrm{N}-\mathrm{NO}_{\mathrm{x}}=12\right)$. The specific rate of denitritation was $0.07 \mathrm{~kg}$ nitrite. $\mathrm{kg}$ volatile suspended solids (VSS)* day after 4.33 $\mathrm{h}$ of anoxic reaction in tests without the addition of cane molasses and 0.19 and $0.12 \mathrm{~kg}$ nitrite. $\mathrm{kg}^{-1}$ VSS*day in the tests with the addition of cane molasses, providing a ratio $\mathrm{BOD} / \mathrm{N}-\mathrm{NO}_{x} 4$ and 14 , respectively. Widening the anoxic reaction time to $22 \mathrm{~h}$ by performing tests on ratio $\mathrm{BOD} / \mathrm{N}-\mathrm{NOx} 12$, the specific rate of denitritation was increased to 0.55 $\mathrm{kg}$ nitrite. $\mathrm{kg}^{-1} \mathrm{VSS}^{*}$ day.
\end{abstract}

Keywords: leachate; nitrite; denitrification; cane molasses; sequencing batch reactor.

\section{INTRODUÇÃO}

O crescimento populacional aliado à crescente demanda por produtos industrializados tem provocado, como consequência maior, a geração contínua de resíduos sólidos que em grande parte são encaminhados a aterros sanitários. Os aterros sanitários possuem como inconveniente a geração de lixiviados de alto potencial de contaminação ambiental (LANGE et al., 2006).

Um dos compostos químicos presentes em maior concentração em lixiviados brutos de aterros sanitários é o nitrogênio amoniacal, sendo a principal forma nitrogenada encontrada no mesmo (BUTKOVSKYI, 2009; GOMES, 2009; RODRIGUES, 2007).
O nitrogênio, apesar de essencial para a manutenção da vida através do ciclo biogeoquímico, deve ser controlado, pois em concentrações excessivas poderá causar problemas como toxicidade, mortandade de peixes e eutrofização de corpos aquáticos (VON SPERLING et al., 2009).

Para atender aos padrões de lançamento de efluentes cada vez mais restritivos, torna-se necessária a busca contínua de melhorias nos processos de tratamento de lixiviados de aterros sanitários existentes, seja através do aperfeiçoamento ou desenvolvimento de tecnologias que aprimorem os sistemas de tratamento já existentes (MARINGONDA JUNIOR, 2008; RODRIGUES, 2007).

口-

'Bacharel em Química Têxtil pela Fundação Universidade Regional de Blumenau (FURB). Mestre em Engenharia Ambiental na FURB - Blumenau (SC), Brasil. ${ }^{2}$ Engenheiro sanitarista. Doutor em Engenharia Ambiental pela Universidade Federal de Santa Catarina (UFSC). Professor do Departamento de Engenharia de Produção e Design da FURB, Professor HV-O1 do SENAI Blumenau - Blumenau (SC), Brasil.

${ }^{3}$ Engenheiro civil. Doutor em Física e Química Ambiental pelo Institut National Polytechinique de Toulouse. Professor do Departamento de Engenharia Civil da FURB Blumenau (SC), Brasil.

Endereço para correspondência: Luana Zilz - Rua São Paulo, 3.250 - 89030-000 - Blumenau (SC), Brasil - E-mail: lu_zilz@hotmail.com

Recebido: 16/02/12 - Aceito: 28/10/13 - Reg. ABES: 234 
Dentre os processos de tratamento existentes, os processos biológicos de remoção de nitrogênio têm sido na sua maior parte adotados para o tratamento de lixiviados de aterros sanitários (LANGE et al., 2006).

No tratamento biológico, o nitrogênio amoniacal é transformado a nitrito e nitrato nas reações de nitrificação, sob a ação de bactérias autotróficas em meio com disponibilidade de oxigênio dissolvido. Os nitratos e/ou nitritos gerados são então desnitrificados a nitrogênio elementar em meio anóxico pela ação de bactérias heterotróficas. Para que essa reação aconteça, ocorre a necessidade de disponibilidade de matéria orgânica (demanda bioquímica de oxigênio - DBO) doadora de elétrons. (AUN, 2007; CARRERA; VICENT; LAFUENTE, 2003).

A fração correspondente à matéria orgânica facilmente biodegradável em lixiviados de aterros sanitários é dependente da fase de degradação dos resíduos, tipo de resíduos aterrados, forma de deposição, assim como das condições climáticas existentes no local. De uma forma geral, a matéria orgânica degradável vai decrescendo em concentração de acordo com a idade do aterro, prevalecendo compostos inertes recalcitrantes, reduzindo desta maneira a razão DBO/DQO (demanda bioquímica de oxigênio/demanda química de oxigênio); sendo por vezes necessária a adição de uma fonte externa de carbono para que o processo de desnitrificação seja realizado completamente (BUTKOVSKYI, 2009; FREITAS, 2009).

Várias fontes de carbono podem ser utilizadas para aumentar a eficiência da desnitrificação como: metanol, etanol, glicose, acetato e melaço de cana. O melaço de cana é um subproduto da produção de açúcar demerara (tipo exportação) e de açúcar cristal (GOPAL; KAMMEN, 2009). É o principal subproduto da indústria açucareira, originado na proporção de 40 a $60 \mathrm{~kg} /$ tonelada de cana processada (PIACENTE, 2005). A reação que expressa a desnitrificação com esta fonte de carbono pode ser esquematizada como (Equação 1):

$\mathrm{C}_{12} \mathrm{H}_{22} \mathrm{O}_{11}+9,6 \mathrm{NO}_{3}^{-}+9,6 \mathrm{H}^{+} \rightarrow 12 \mathrm{CO}_{2}+4,8 \mathrm{~N}_{2}+15,8 \mathrm{H}_{2} \mathrm{O}$

Ultimamente, tem havido interesse crescente na nitrificação e desnitrificação via nitrito, pois de acordo com o balanço estequiométrico o fornecimento de oxigênio dissolvido para as bactérias autotróficas converterem amônia a nitrito é menor, assim como a quantidade de matéria orgânica doadora de elétrons para a desnitrificação (QUEIROZ et al. 2011; ZAFARZADEH et al., 2011). A desnitrificação via nitrito requer um consumo $25 \%$ menor de oxigênio dissolvido para a oxidação do nitrogênio amoniacal e $40 \%$ menor de fonte de carbono para a desnitrificação do nitrito gerado (SPAGNI; MARSILI-LIBELLI; LAVAGNOLO, 2008). A nitrificação é uma reação de oxidação do nitrogênio amoniacal, sendo requerida a presença de oxigênio dissolvido. O oxigênio dissolvido é utilizado para a respiração de bactérias autotróficas que consomem alcalinidade do lixiviado bruto, além de compostos inorgânicos como $\mathrm{CO}_{2}(\mathrm{AHN}, 2006)$. A nitrificação é uma reação de duas etapas em que bactérias oxidadoras de amônia do gênero Nitrossomas transformam a amônia a nitrito (nitritação) e bactérias oxidadoras de nitrito do gênero Nitrobacter transformam nitrito a nitrato (nitratação). Conforme Butkovskyi (2009) a nitrificação pode ser esquematizada da seguinte forma (Equação 2):

$\mathrm{NH}_{4}^{+}+\frac{3}{2} \mathrm{O}_{2} \rightarrow \mathrm{NO}_{2}^{-}+\mathrm{H}_{2} \mathrm{O}+2 \mathrm{H}^{+}$

$\mathrm{NO}_{2}^{-}+\frac{1}{2} \mathrm{O}_{2} \rightarrow \mathrm{NO}_{3}^{-}$

$\mathrm{NH}_{4}^{+}+2 \mathrm{O}_{2} \rightarrow \mathrm{NO}_{3}^{-}+\mathrm{H}_{2} \mathrm{O}+2 \mathrm{H}^{+}$

De acordo com Freitas (2009) vários fatores podem afetar a nitrificação, levando ao acúmulo de nitrito no reator nitrificante. Parâmetros como $\mathrm{pH}$, temperatura e oxigênio dissolvido podem ser algumas das causas de acúmulo de nitrito se não operadas adequadamente. Em valores de $\mathrm{pH}$ abaixo de 6,0, a atividade das bactérias nitrificantes é inibida (CAMPOS et al., 2007) e, em temperaturas acima de $20^{\circ} \mathrm{C}$, a reação de nitrificação ocorre mais lentamente, levando ao acúmulo de nitrito. Além disso, concentrações de oxigênio dissolvido abaixo de 2,0 mg. $\mathrm{L}^{-1}$ reduzem a ação das bactérias oxidadoras de nitrito. Outros fatores como a presença de amônia livre também afetam negativamente a reação de nitrificação a nitrato, sendo que sob concentração de amônia livre de 0,1 a $10 \mathrm{mg} \cdot \mathrm{L}^{-1}$ há a inibição da atividade das Nitrossomonas e sob concentração de 10 a 150 mg.L.-1 ocorre a inibição da atividade das Nitrobacter.

A desnitrificação é a reação de redução de nitrato a nitrogênio gasoso em meio anóxico utilizando-se matéria orgânica biodegradável como fonte de energia (AHN, 2006). A redução de formas oxidadas de nitrogênio pode ser realizada por Pseudomonas fluorescens, Pseudomonas denitrificans, denitrificans Paracoccus e Micrococcus denitrificans, e outras bactérias (ESTUARDO et al., 2008). A oxidação da matéria orgânica e a desnitrificação via nitrato e via nitrito podem ser esquematizadas de acordo com as Equações 3 a 5 (VAN HAANDEL; KATO; VON SPERLING, 2009), sendo a matéria orgânica doadora de elétrons para a redução das formas oxidadas de nitrogênio.

Oxidação da matéria orgânica:

$\mathrm{C}_{x} \mathrm{H}_{y} \mathrm{O}_{z}+(2 x-z) \mathrm{H}_{2} \mathrm{O} \rightarrow x \mathrm{CO}_{2}+(4 x+y-2 z) \mathrm{H}^{+}+(4 x+y-2 z) e$

onde desnitrificação via nitrato: 
$e+\frac{6}{5} \mathrm{H}^{+}+\frac{1}{5} \mathrm{NO}_{3}^{-} \rightarrow \frac{1}{10} \mathrm{~N}_{2}+\frac{1}{5} \mathrm{H}_{2} \mathrm{O}$

e desnitrificação via nitrito:

$e+\frac{4}{3} \mathrm{H}^{+}+\frac{1}{3} \mathrm{NO}_{2}^{-} \rightarrow \frac{1}{6} \mathrm{~N}_{2}+\frac{2}{3} \mathrm{H}_{2} \mathrm{O}$

Na desnitrificação as principais variáveis interferentes na eficiência do processo são o $\mathrm{pH}$, o oxigênio dissolvido e a presença de substâncias tóxicas (VON SPERLING, 1997). As condições de $\mathrm{pH}$ mais favoráveis para a desnitrificação situam-se entre 7,0 e 9,0. Por sua vez, o meio deve estar ausente de oxigênio fornecendo condições anóxicas para que as bactérias heterotróficas utilizem o oxigênio dos nitritos e nitratos para a respiração e conversão a nitrogênio elementar (AHN, 2006). Além disso, a eficiência da desnitrificação é também influenciada pela concentração de matéria orgânica biodegradável e pela temperatura de operação do efluente no reator. O nitrito é um produto intermediário da desnitrificação e dependendo do $\mathrm{pH}$ e da temperatura do reator pode prevalecer na forma ionizada ou não-ionizada.

Ao longo do tempo a razão $\mathrm{C} / \mathrm{N}$ de aterros sanitários diminui devido à menor proporção de compostos orgânicos biodegradáveis presentes nos lixiviados, podendo afetar desta forma a desnitrificação. Nos casos em que o próprio efluente bruto não é capaz de desnitrificar totalmente os nitritos e/ou nitratos formados na fase aeróbia podem ser adicionadas fontes externas de carbono (AHN, 2006).

A escolha da fonte de carbono externa a ser utilizada nos processos biológicos é fundamental na concepção de instalações de tratamento de águas residuárias, principalmente quando se objetiva alcançar melhores resultados em termos de eficiência. Nesta tomada de decisão, deverão ser avaliados conjuntamente os custos, a disponibilidade do substrato e a taxa de desnitrificação de nitratos e nitritos, como também a garantia de fornecimento contínuo, sendo que esses requerimentos podem ser obedecidos utilizando-se subprodutos industriais (CARRERA; VICENT; LAFUENTE, 2003).

O presente trabalhou avaliou em escala laboratorial a utilização de melaço de cana-de-açúcar, um substrato da produção do açúcar, como fonte externa alternativa de carbono, objetivando aumentar a eficiência da desnitrificação de um sistema tratando lixiviado de aterro sanitário operado sob acúmulo de nitrito.

\section{METODOLOGIA}

\section{Caracterização química do lixiviado de aterro sanitário em estudo}

O lixiviado bruto foi coletado em um aterro sanitário antigo, em operação por aproximadamente 22 anos, localizado em Biguaçu- região litorânea de Santa Catarina. Dentre as técnicas utilizadas para o tratamento do lixiviado no local, tem-se a nitrificação com pré-desnitrificação em sistema de lodos ativados. O lixiviado bruto foi caracterizado quimicamente em triplicata para as variáveis: alcalinidade total, $\mathrm{DBO}_{5}$, DQO, $\mathrm{pH}$ e nitrogênio nas formas de nitrato, nitrito, amoniacal e total. Os procedimentos adotados foram baseados no Standard Methods for Examination of Water and Wastewater (2005).

\section{Desnitrificação em escala laboratorial}

Primeiramente, o inóculo foi coletado do reator nitrificante da estação de tratamento do lixiviado e aclimatado no lixiviado bruto por um período de quatro dias, com adição progressiva do lixiviado na proporção de 0,03 a 0,33 L lixiviado. $\mathrm{L}^{-1}$ inóculo. Após o processo de aclimatação, foram realizados ensaios de desnitrificação em escala laboratorial em um reator em batelada sequencial (RBS) de polipropileno e volume útil de $2 \mathrm{~L}$ com ciclos de três repetições para cada razão $\mathrm{DBO} / \mathrm{N}-\mathrm{NO}_{\mathrm{x}}$, aplicando-se melaço de cana como fonte externa de carbono. Cada ciclo de desnitrificação consistiu das seguintes fases: alimentação $(0,25 \mathrm{~h})$, com adição de lixiviado bruto ao reator, reação anóxica $(4,33 \mathrm{~h}$ para as razões $\mathrm{DBO} / \mathrm{N}$ $\mathrm{NO}_{\mathrm{x}}=0,37 ; 4$ e 14), sob $40 \mathrm{rpm}$, sedimentação (1 h) e descarte $(0,25 \mathrm{~h})$, mesmo procedimento adotado por Butkovskyi (2009). Com a finalidade de avaliar a influência do tempo de detenção hidráulica (TDH), também realizaram-se ensaios de desnitrificação sob razão DBO/N-NO igual a 12 e tempo de fase anóxica total de $22 \mathrm{~h}$. Anteriormente ao período anóxico, adicionou-se $1.000 \mathrm{mg} . \mathrm{L}^{-1}$ de nitrito sintético para padronizar a concentração de nitrito a ser desnitrificada (Figura 1). Os ensaios sem adição de melaço apresentaram razão $\mathrm{DBO} / \mathrm{N}-\mathrm{NO}_{\mathrm{x}}$ igual a 0,37. A quantidade de melaço de cana adicionada como fonte externa alternativa de carbono foi variada, fornecendo razão $\mathrm{DBO} / \mathrm{N}-\mathrm{NO}_{\mathrm{x}}$ de 4, 12, e 14. Essa variação na quantidade aplicada de melaço de cana ao reator visou avaliar a possibilidade de uso desta fonte de carbono para ampliar a eficiência de desnitrificação de um reator sob acúmulo de nitrito.

Os parâmetros DQO, nitrogênio amoniacal $\left(\mathrm{N}-\mathrm{NH}_{3}\right)$, nitrato $\left(\mathrm{N}-\mathrm{NO}_{3}\right)$, nitrito $\left(\mathrm{N}-\mathrm{NO}_{2}\right)$, e sólidos suspensos voláteis (SSV), foram analisados duas vezes por semana para cada razão $\mathrm{DBO} / \mathrm{N}-\mathrm{NO}_{\mathrm{x}}$. As amostras foram coletadas e acondicionadas sob refrigeração a $4^{\circ} \mathrm{C}$ como método de preservação das amostras. As coletas de amostras obedeceram aos seguintes intervalos de tempo (Tabela 1).

\section{RESULTADOS E DISCUSSÃO}

\section{Caracterização química do lixiviado bruto em estudo}

O lixiviado bruto coletado apresentou características químicas de lixiviado de aterro sanitário antigo (Tabela 2). O conteúdo de matéria orgânica biodegradável foi muito baixo $\left(\mathrm{DBO}_{5}=158 \mathrm{mg} \cdot \mathrm{L}^{-1}\right.$ e $\left.\mathrm{DQO}=2137 \mathrm{mg} \cdot \mathrm{L}^{-1}\right)$. A baixa razão $\mathrm{DBO}_{5} / \mathrm{DQO}$ e $\mathrm{pH}$ foram 


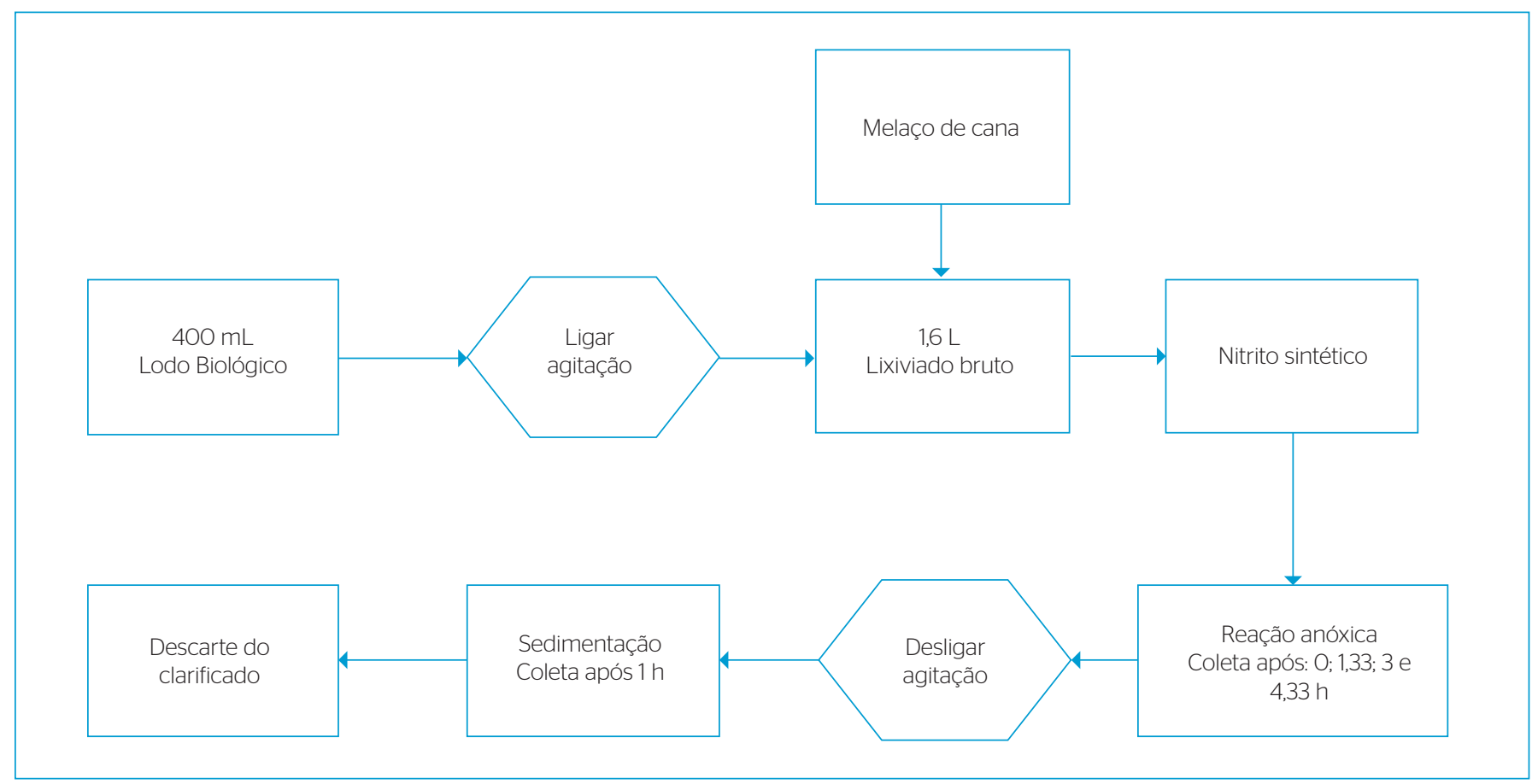

Figura 1 - Operação do reator em batelada sequencial em escala laboratorial.

Tabela 1 - Intervalos de tempo de reação anóxica para coleta de amostras.

\begin{tabular}{c|c|c|c|c|c}
\hline Razão DBO/N-NO & $\mathrm{t}=0 \mathrm{~h}$ & $\mathrm{t}=1,33 \mathrm{~h}$ & $\mathrm{t}=3 \mathrm{~h}$ & $\mathrm{t}=4,33 \mathrm{~h}$ & $\mathrm{t}=22 \mathrm{~h}$ \\
\hline $\mathrm{O}, 37$ & $\mathrm{X}$ & $\mathrm{X}$ & $\mathrm{X}$ & $\mathrm{X}$ & \\
\hline 4 & $X$ & $X$ & $X$ & $X$ & \\
\hline 12 & $X$ & $X$ & $X$ & $X$ & $X$ \\
\hline 14 & $X$ & $X$ & $X$ & $X$ & \\
\hline
\end{tabular}

Para a análise de sólidos suspensos voláteis, coletaram-se amostras no início dos ciclos experimentais (Ciclo I, t=0 h) e após término da reação anóxica no quarto ciclo experimental (Ciclo IV, $\mathrm{t}=4,33 \mathrm{~h}$ para $\mathrm{DBO} / \mathrm{N}-\mathrm{NO}_{\mathrm{x}} \mathrm{O}, 37 ; 4 \mathrm{e} 14 \mathrm{e} \mathrm{t}=22 \mathrm{~h}$ para $\mathrm{DBO} / \mathrm{N}-\mathrm{NO}_{x}$ 12). Amostras também foram coletadas após o período de sedimentação para todos os parâmetros analisados.

DBO: demanda bioquímica de oxigênio.

Tabela 2 - Caracterização química do lixiviado bruto.

\begin{tabular}{|c|c|c|c|}
\hline Parâmetro & $\begin{array}{l}\text { Concentração } \\
\text { média } \\
\left(\mathrm{mg} \cdot \mathrm{L}^{-1}\right)\end{array}$ & $\begin{array}{l}\text { Concentração } \\
\text { máxima } \\
\left(m g \cdot \mathrm{L}^{-1}\right)\end{array}$ & $\begin{array}{l}\text { Concentração } \\
\text { mínima } \\
\left(\mathrm{mg} \cdot \mathrm{L}^{-1}\right)\end{array}$ \\
\hline Alcalinidade total & 9.470 & 10.000 & 8.800 \\
\hline $\mathrm{DBO}_{5,20^{\circ} \mathrm{C}}$ & 158 & 475 & $\mathrm{O}$ \\
\hline DQO & 2.137 & 2.204 & 2.093 \\
\hline $\mathrm{DBO}_{5} / \mathrm{DQO}$ & 0,08 & 0,22 & O \\
\hline Nitrogênio amoniacal & 2.937 & 3.030 & 2.770 \\
\hline $\begin{array}{l}\text { Nitrogênio na forma } \\
\text { de nitrato }\end{array}$ & 22,4 & 38,6 & 14,0 \\
\hline $\begin{array}{l}\text { Nitrogênio na forma } \\
\text { de nitrito }\end{array}$ & 2,5 & 5,1 & 0,6 \\
\hline Nitrogênio total & 3.783 & - & - \\
\hline $\mathrm{pH}$ & 9,0 & 9,1 & 8,9 \\
\hline
\end{tabular}

DBO: demanda bioquímica de oxigênio; DQO: demanda química de oxigênio. consistentes com os estudos realizados por Tengrui et al. (2007) que obtiveram razão $\mathrm{DBO}_{5} / \mathrm{DQO}$ baixa $(0,05)$ e $\mathrm{pH}$ na faixa de $8,0-9,0$ para lixiviado antigo. A maior parte no nitrogênio total encontrado está na forma de nitrogênio amoniacal (77\%), sendo esta espécie química responsável pela concentração de $\mathrm{DBO}_{5}$ necessária para a completa desnitrificação dos nitratos e nitritos gerados na nitrificação.

\section{Desnitrificação com lixiviado bruto como fonte de carbono e sem adição de melaço de cana $\left(\mathrm{DBO}_{5} / \mathrm{N}\right.$ - $\mathrm{NO}_{\mathrm{x}}=0,37$ e tempo total de fase anóxica de 4,33 h)}

O maior consumo de DQO ocorreu durante a fase de sedimentação no primeiro ciclo experimental com consumo de $1.532 \mathrm{mg} . \mathrm{L}^{-1}$ de DQO correspondendo a uma eficiência de remoção de 34\%. Spagni, MarsiliLibelli e Lavagnolo (2008) obtiveram eficiências de remoção de DQO de 30 a 40\% estudando a desnitrificação de nitrito com lixiviado de aterro sanitário antigo como fonte de carbono em RBS.

Maringonda Junior (2008) estudou lixiviado de aterro sanitário antigo de $\mathrm{DBO}_{5}$ de 111 mg.L-1 e obteve eficiência de remoção de DQO de 28\%, resultado próximo ao obtido neste estudo. A DQO remanescente deveu-se à presença de compostos recalcitrantes típicos de aterros sanitários antigos.

Após adição do nitrito sintético observou-se que grande porção do reagente converteu-se a nitrato, um resultado inesperado que pode ter ocorrido pela oxidação do reagente devido ao seu comporta-

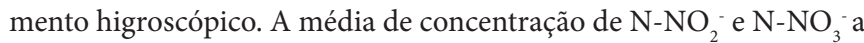
ser reduzida no reator anóxico foi de $667 \mathrm{mg} . \mathrm{L}^{-1}$. Em relação à remoção de nitrato, observou-se diminuição da concentração até 4,33 h de reação anóxica, com posterior aumento de concentração durante a 
fase de sedimentação biológica no ciclo I superando a concentração inicial (Figura 2).

No ciclo IV, observou-se redução da concentração de $\mathrm{N}^{-\mathrm{NO}_{3}}$ - entre 3 e 4,33 h de reação anóxica, com posterior aumento de concentração durante a fase de sedimentação. Uma hipótese que poderia explicar a concentração de $\mathrm{N}-\mathrm{NO}_{3}$ - durante a fase de sedimentação superar a concentração inicial nos ciclos I e IV é a oxidação anaeróbia de amônia, processo conhecido como Annamox, sendo por esse motivo que nos ensaios com adição de melaço passou-se a analisar o parâmetro nitrogênio amoniacal. Ahn (2006) explicou em seu trabalho que no processo Annamox micro-organismos utilizam nitrito e/ou nitrato como elétron aceptor para oxidar o nitrogênio amoniacal.

Não foi possível obter a concentração padrão de $1.000 \mathrm{mg} \cdot \mathrm{L}^{-1}$ de nitrogênio na forma de nitrito para avaliar a desnitrificação. Observou-se pequena redução na concentração de $\mathrm{N}-\mathrm{NO}_{2}$ - após 1,33 e 4,33 h de fase anóxica nos ciclos I e IV, respectivamente, correspondendo a uma eficiência de redução de 5 e 12\%. Maringonda Junior (2008) observou que a $\mathrm{DBO}_{5}$ de um lixiviado de aterro sanitário antigo não foi suficiente para realizar a desnitrificação completa das formas oxidadas de nitrogênio.

Assim como ocorreu com o nitrogênio na forma de nitrato, observou-se que a concentração de nitrogênio na forma de nitrito foi maior durante a fase de sedimentação com concentração superior a obtida no estado inicial, podendo ser devido à oxidação do nitrogênio amoniacal, mesma hipótese proposta para o nitrogênio na forma de nitrato (Figura 3). Como forma de provar tal hipótese, o parâmetro nitrogênio amoniacal passou a ser analisado nos ensaios com adição de melaço de cana.

A desnitrificação pôde ser observada após 1,33 h de fase anóxica no ciclo I e após 4,33 h de fase anóxica no ciclo IV. No ciclo IV, $38,5 \mathrm{mg} . \mathrm{L}^{-1}$ de $\mathrm{N}-\mathrm{NO}_{\mathrm{x}}$ foram reduzidos, mas com aumento de concentração das formas nitrogenadas oxidadas $\left(\mathrm{N}^{-\mathrm{NO}_{2}}{ }_{2}^{-} \mathrm{e} \mathrm{N}-\mathrm{NO}_{3}{ }^{-}\right)$durante a sedimentação (Figura 4). A taxa específica de desnitritação foi de $0,07 \mathrm{~kg}$ nitrito. $\mathrm{kg}^{-1} \mathrm{SSV}^{\star}$ dia após 4,33 h de fase anóxica no ciclo IV.

Em relação ao SSV, o sistema apresentou perda de biomassa após o quarto ciclo experimental (12\%), reduzindo de $3055 \mathrm{mg} \cdot \mathrm{L}^{-1}$ de biomassa inicial para $2675 \mathrm{mg} . \mathrm{L}^{-1}$, talvez tendo ocorrido lavagem da biomassa do sistema. Quanto ao índice volumétrico de lodo (IVL), o lodo apresentou boa decantabilidade e adensamento, com o lodo biológico ocupando um volume de 53,5 mL.g-1 SSV após o quarto ciclo experimental.

\section{Desnitrificação com lixiviado bruto + melaço de cana como fonte de carbono $\left(\mathrm{DBO}_{5} / \mathrm{N}-\mathrm{NO}_{\mathrm{x}}=4\right.$ e 14 com tempo total de fase anóxica $=4,33 \mathrm{~h}$ e $\mathrm{DBO}_{5} / \mathrm{N}$ - $\mathrm{NO}_{\mathrm{x}}=12 \mathrm{com}$ tempo total de fase anóxica de $22 \mathrm{~h}$ )}

Nos ensaios realizados com adição de melaço de cana como fonte externa alternativa de carbono observou-se redução no consumo de DQO para desnitrificar nitrito e nitrato à medida que se aumentou $\mathrm{a}$ dosagem de melaço. A eficiência máxima de redução alcançada foi de

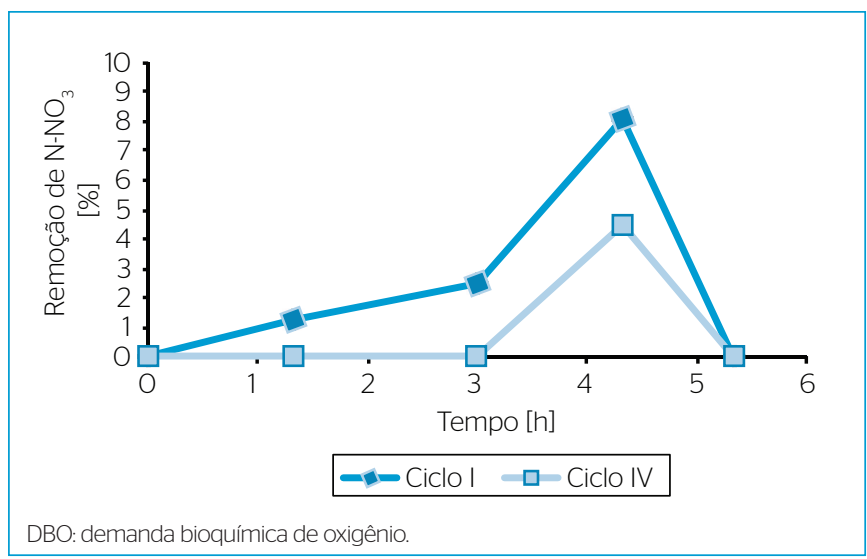

Figura 2 - Variação da concentração de nitrogênio na forma de nitrato para razão $\mathrm{DBO}_{5} / \mathrm{N}-\mathrm{NO}_{\mathrm{x}}=0,37$.

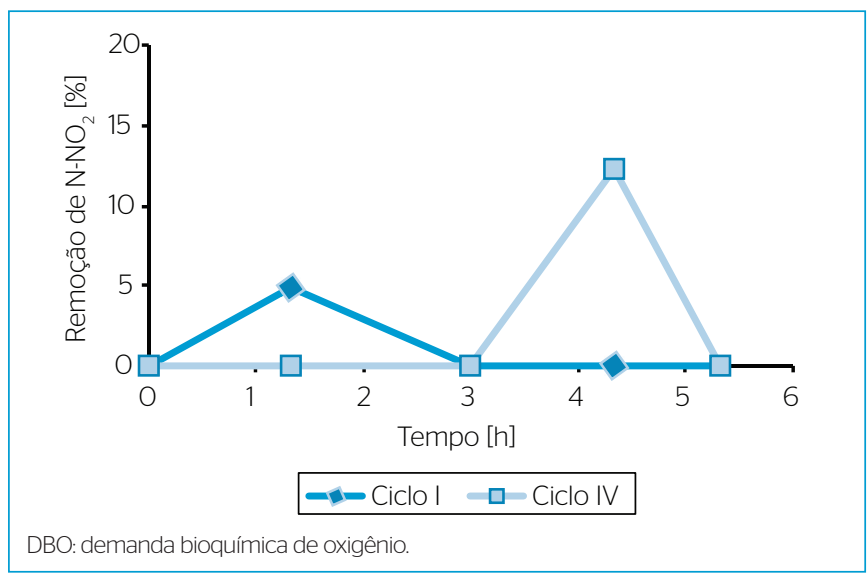

Figura 3 - Variação da concentração de nitrogênio na forma de nitrito para razão $\mathrm{DBO} / \mathrm{N}-\mathrm{NO}_{\mathrm{x}}=0,37$.

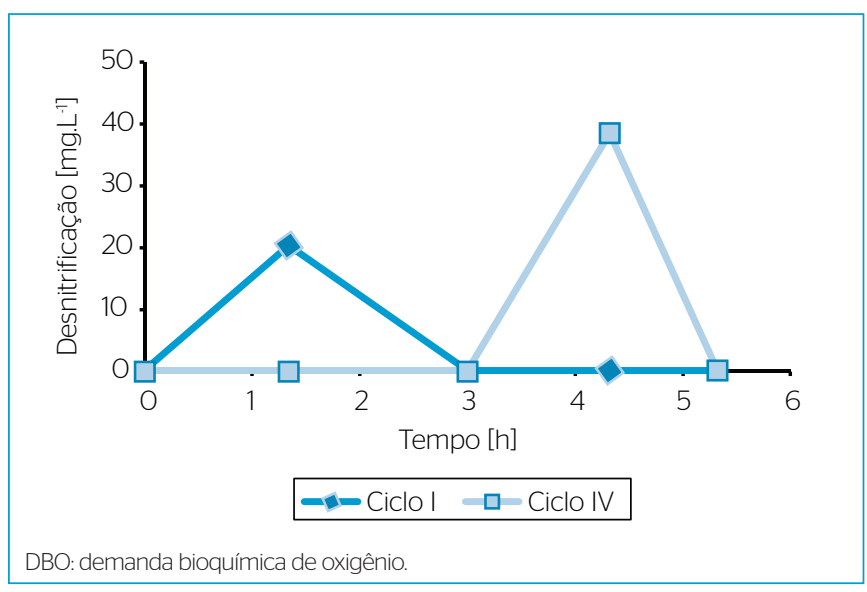

Figura 4 - Desnitrificação de nitrogênio na forma de nitrato e nitrito sob razão $\mathrm{DBO} / \mathrm{N}-\mathrm{NO}_{\mathrm{x}}=0,37$.

$69 \%$ e $30 \%$, correspondendo a um consumo de 1.282 e 3.290 mg.L $\mathrm{L}^{-1}$ durante a sedimentação no ciclo IV sob razão $\mathrm{DBO}_{5} / \mathrm{N}-\mathrm{NO}_{\mathrm{x}}$ de $4 \mathrm{e}$ 14 e tempo de fase anóxica total de 4,33 h. Nos ensaios conduzidos sob razão $\mathrm{DBO}_{5} / \mathrm{N}-\mathrm{NO}_{\mathrm{x}}$ de 12 , a eficiência máxima de remoção de 
DQO foi de 58\% também após a sedimentação biológica. A diminuição na eficiência de remoção de DQO com o aumento da quantidade de melaço adicionada poderia ser explicada pelo fato de que o melaço fornece uma maior concentração de matéria orgânica sendo que, provavelmente, parte dessa matéria não é absorvida pela microbiota existente no reator.

Nos ensaios realizados sob razão $\mathrm{DBO} / \mathrm{N}-\mathrm{NO}_{x}$ de 4 houve redução da concentração de nitrogênio na forma de nitrato na primeira hora anóxica nos ciclos I e IV e durante a sedimentação biológica no ciclo I, com redução máxima observada de 8,8\% após 1,33 h de fase anóxica no ciclo I. Em relação à remoção das formas nitrogenadas oxidadas não se observou remoção de nitrogênio na forma de nitrato em relação à concentração inicial nos ensaios realizados sob razão $\mathrm{DBO} / \mathrm{N}-\mathrm{NO}_{\mathrm{x}}$ de 14 . Nos ensaios realizados sob razão $\mathrm{DBO} / \mathrm{N}-\mathrm{NO}_{\mathrm{x}}$ de 12 e tempo de fase anóxica de $22 \mathrm{~h}$, observou-se remoção de $\mathrm{N}-\mathrm{NO}_{3}$ - após $1,33 \mathrm{~h}$ de fase anóxica e entre 4,33 e 22 h de fase anóxica e na sedimentação nos ciclos I e IV, assim como após $3 \mathrm{~h}$ de fase anóxica no ciclo I. A eficiência máxima de remoção de nitrogênio na forma de nitrato foi de $54,7 \%$ após 4,33 h e 60,9\% após 22 h de fase anóxica. Durante os ensaios notou-se também variação negativa das concentrações de nitrogênio na forma de nitrato sob diferentes tempos de fase anóxica nos ensaios realizados sob diferentes razões $\mathrm{DBO} / \mathrm{N}-\mathrm{NO}_{\mathrm{x}}$. Isto foi devido à oxidação anaeróbia de nitrogênio amoniacal (Annamox) presente no afluente bruto e, também, à própria oxidação do reagente sintético utilizado.

Quanto ao nitrogênio na forma de nitrito, observou-se maior eficiência de remoção no quarto ciclo experimental em todos os ensaios realizados sob adição de melaço de cana como fonte alternativa de carbono. À medida que se aumentou a concentração de melaço no reator, a taxa de remoção de nitrogênio na forma de nitrito foi ampliada. As eficiências máximas de remoção foram de $8,6 \%$ após $3 \mathrm{~h}$ nos ensaios realizados sob razão $\mathrm{DBO} / \mathrm{N}-\mathrm{NO}_{\mathrm{x}}$ de 4; $30,8 \%$ após $22 \mathrm{~h}$ operando-se sob razão $\mathrm{DBO} / \mathrm{N}-\mathrm{NO}_{\mathrm{x}}$ de 12 e $35,4 \%$ após 1,33 h sob razão $\mathrm{DBO} / \mathrm{N}-\mathrm{NO}_{\mathrm{x}}$ de 14 . A ampliação do tempo de fase anóxica total de $4,33 \mathrm{~h}$ para $22 \mathrm{~h}$ auxiliou positivamente na eficiência da taxa de remoção de nitrogênio na forma de nitrato e de nitrito nos experimentos conduzidos sob razão $\mathrm{DBO} / \mathrm{N}-\mathrm{NO}_{\mathrm{x}}$ de 12 (Figura 5).

Ocorreu aumento de concentração de nitrogênio na forma de nitrito após 1,33 h e entre 3 h e 4,33 h de reação anóxica no ciclo I, e também durante a sedimentação do lodo biológico nos ensaios realizados sob razão $\mathrm{DBO} / \mathrm{N}-\mathrm{NO}_{\mathrm{x}}$ de 12 e tempo de fase anóxica total de $22 \mathrm{~h}$.

O aumento da concentração de nitrogênio na forma de nitrito após 1,33 h de fase anóxica no ciclo I deveu-se a desnitrificação parcial de nitrogênio na forma de nitrato. $\mathrm{O}$ aumento da concentração de nitrogênio na forma de nitrito e de nitrato após $4,33 \mathrm{~h}$ de fase anóxica no ciclo I deveu-se à oxidação de nitrogênio amoniacal. Da mesma forma que ocorreu na primeira hora anóxica, o aumento da concentração de nitrogênio na forma de nitrito durante a sedimentação deveu-se ao fato do nitrogênio na forma de nitrato ter desnitrificado parcialmente.

O somatório das concentrações das formas oxidadas de nitrogênio ao início dos experimentos utilizando-se melaço de cana foi inferior ao obtido nos ensaios realizados sem adição de fonte alternativa de carbono, demonstrando a dificuldade em padronizar a concentração de nitrito com o reagente sintético utilizado. Percebeu-se grande variação da concentração de nitrogênio amoniacal no sistema experimental, ocorrendo oxidação anaeróbia de amônia a nitrogênio gasoso na presença de nitrato e nitrito, assim como a oxidação do mesmo a nitrito e nitrato ao longo da fase anóxica, dadas as concentrações maiores em relação ao estado inicial

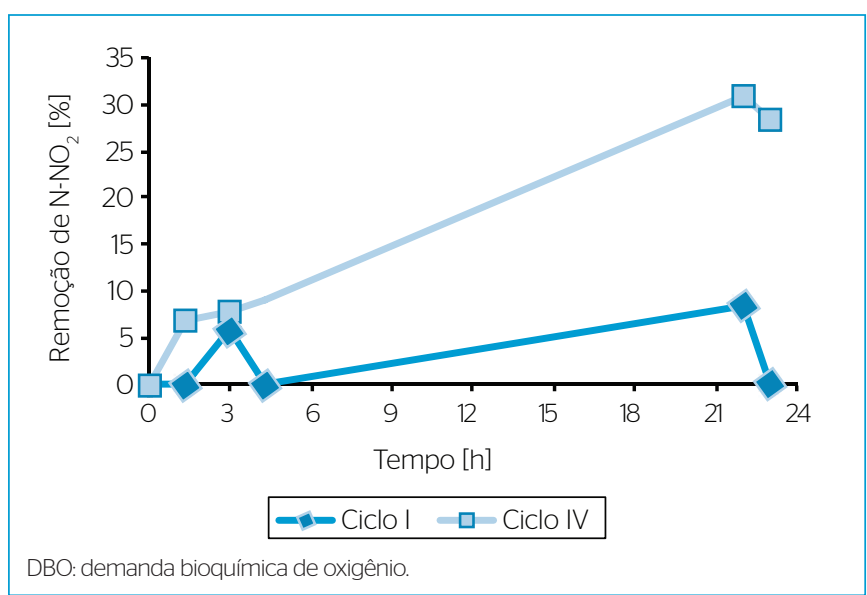

Figura 5 - Remoção de nitrogênio na forma de nitrito nos ensaios realizados sob razão $\mathrm{DBO} / \mathrm{N}-\mathrm{NO}_{\mathrm{x}}=12$ e tempo de fase anóxica total de $22 \mathrm{~h}$.

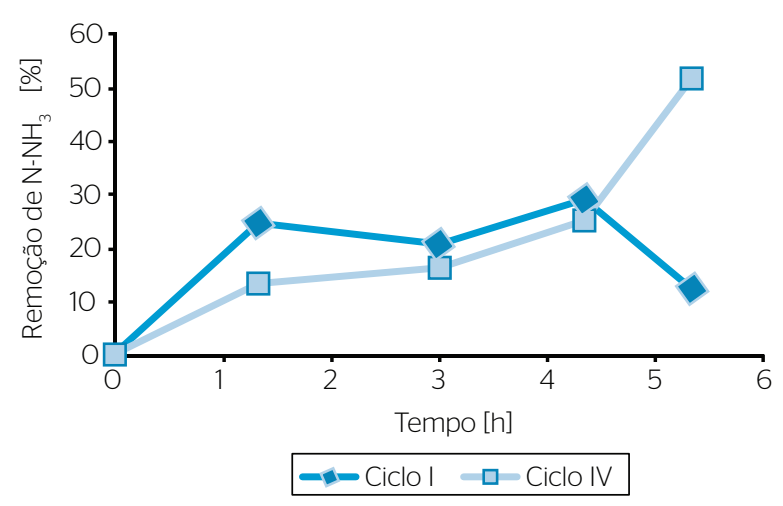

DBO: demanda bioquímica de oxigênio.

Figura 6 - Remoção de nitrogênio amoniacal nos ensaios realizados sob razão $\mathrm{DBO} / \mathrm{N}-\mathrm{NO}_{\mathrm{x}}=14$. 
(Figura 6). A eficiência máxima de remoção de nitrogênio amoniacal foi de 16 e $52 \%$ durante a sedimentação nos ciclos I e IV, respectivamente nos ensaios executados sob razão $\mathrm{DBO} / \mathrm{N}-\mathrm{NO}_{\mathrm{x}}$ de 4 e 14. Nos ensaios realizados sob razão DBO/N-NO de 12 e tempo de fase anóxica total de $22 \mathrm{~h}$, não se observou parcela elevada de remoção de nitrogênio amoniacal do sistema, ocorrendo eficiência máxima de remoção durante a sedimentação, após 22 $\mathrm{h}$ de fase anóxica. A ampliação do tempo de fase anóxica foi responsável pela remoção de nitrogênio amoniacal do sistema, visto após 4,33 h de reação anóxica não haver ocorrido remoção desse parâmetro em ambos os ciclos.

Nos ensaios realizados sob DBO/N-NO de 4, observa-se a desnitrificação durante a primeira hora anóxica nos ciclos I e IV ocorrendo desnitrificação de nitrogênio amoniacal e de nitrogênio na forma de nitrato e de nitrito também durante a sedimentação biológica, podendo a desnitrificação ter ocorrido devido ao processo Annamox. Foram desnitrificados na primeira hora anóxica $19,7 \mathrm{mg} \mathrm{N}-\mathrm{NO}_{\mathrm{x}} \cdot \mathrm{L}^{-1} \mathrm{e} 5 \mathrm{mg} \mathrm{N}-\mathrm{NO}_{\mathrm{x}} \cdot \mathrm{L}^{-1}$ nos ciclos I e IV. Ocorre porém mais intensamente essa reação durante a sedimentação com $30,7 \mathrm{mg}$ $\mathrm{N}-\mathrm{NO}_{\mathrm{x}} \cdot \mathrm{L}^{-1} \mathrm{e} 18,3 \mathrm{mg} \mathrm{N}-\mathrm{NO}_{\mathrm{x}} \cdot \mathrm{L}^{-1}$ desnitrificados nos ciclos I e IV. A taxa específica de desnitritação para esses ensaios foi de $0,19 \mathrm{~kg} \mathrm{~N}-\mathrm{NO}_{2} \cdot \mathrm{kg}^{-1}$ $\mathrm{SSV}^{\star}$ dia. Houve desnitrificação de $26 \mathrm{mg} \cdot \mathrm{L}^{-1}, 18,33 \mathrm{mg} \cdot \mathrm{L}^{-1} \mathrm{e} 8 \mathrm{mg} \cdot \mathrm{L}^{-1}$ das formas oxidadas, após 1,33 h, 3 h e 5,33 h respectivamente no ciclo IV $\left(\mathrm{DBO} / \mathrm{N}-\mathrm{NO}_{\mathrm{x}}=14\right)$. A desnitrificação ocorreu devido à redução de nitrito após 1,33 h, oxidação de amônia após 3 h e redução de nitrato após 5,33 h no Ciclo IV. Pickbrenner (2002) explica que durante a fase de sedimentação pode ocorrer ainda a desnitrificação. Ele obteve consumo de nitrogênio amoniacal durante a fase anóxica e baixas taxas de desnitrificação. A taxa específica de desnitritação em relação ao estado inicial foi de $0,12 \mathrm{~kg} \mathrm{~N}-\mathrm{NO}_{2} \cdot \mathrm{kg}^{-1} \mathrm{SSV}^{\star}$ dia nos ensaios realizados sob razão $\mathrm{DBO} / \mathrm{N}-\mathrm{NO}_{\mathrm{x}}$ de 14. Comparando-se com o estado inicial foram desnitrificados $26,7 \mathrm{mg} \mathrm{N}-\mathrm{NO}_{\mathrm{x}} \cdot \mathrm{L}^{-1}$ nas primeiras três horas de fase anóxica no ciclo I e 48,7 mg N-NO $\cdot \mathrm{L}^{-1}$ entre 4,33 e $22 \mathrm{~h}$ de fase anóxica nos ensaios sob razão $\mathrm{DBO} / \mathrm{N}-\mathrm{NO}_{\mathrm{x}}$ de 12 . No ciclo IV observou-se redução de 134,7 mg N-NO $\mathrm{N}_{x} \cdot \mathrm{L}^{-1}$ após 1,33 h de fase anóxica e 190,7 mg $\mathrm{N}-\mathrm{NO}_{\mathrm{x}} \cdot \mathrm{L}^{-1}$ entre 4,33 e $22 \mathrm{~h}$ de fase anóxica. A ampliação do tempo de fase anóxica para $22 \mathrm{~h}$ foi responsável pelo aumento da desnitrificação, sendo observada a remoção de 137,3 mg. $\mathrm{L}^{-1}$ de nitrogênio na forma de nitrato e nitrito, correspondendo a um aumento na remoção de $39 \%$. A taxa específica de desnitritação foi de $0,55 \mathrm{~kg} \mathrm{~N}-\mathrm{NO}_{2} \cdot \mathrm{kg}^{-1}$ $\mathrm{SSV}^{\star}$ dia, sendo bem superior aos ensaios anteriores, provavelmente devido à ampliação do tempo de fase anóxica. Os resultados obtidos demonstram que a adição de melaço de cana ampliam a taxa específica de desnitritação.

O IVL mostrou-se também superior quanto maior a parcela de melaço adicionado ao sistema desnitrificante, resultando no decaimento das características de sedimentação do lodo biológico podendo favorecer a lavagem da biomassa. O IVL de $118,8 \mathrm{~mL}\left(\mathrm{mg} \mathrm{SSV}^{-1}\left(\mathrm{DBO} / \mathrm{N}-\mathrm{NO}_{\mathrm{x}}\right.\right.$ de 4$)$ e $157,75 \mathrm{~mL}(\mathrm{mg} \mathrm{SSV})^{-1}$ $\left(\mathrm{DBO} / \mathrm{N}-\mathrm{NO}_{\mathrm{x}}\right.$ de 14$)$ foi bem superior ao encontrado ao se analisar a desnitrificação do lixiviado bruto sem adição de fonte externa de carbono. A análise de SSV resultou na perda de $272 \mathrm{mg} . \mathrm{L}^{-1}$ e $682 \mathrm{mg} . \mathrm{L}^{-1}$ de biomassa do sistema nos ensaios sob razão $\mathrm{DBO} / \mathrm{N}-\mathrm{NO}_{x}$ de 4 e 14 , respectivamente, correspondendo a uma redução de 14 e $34 \%$ em relação ao valor inicial, provavelmente devido à lavagem da biomassa durante a fase de descarte, visto o problema de decantabilidade que ocorreu nesses ensaios. Aumentando-se o tempo de fase anóxica para $22 \mathrm{~h}$ nos ensaios sob razão $\mathrm{DBO} / \mathrm{N}-\mathrm{NO}_{\mathrm{x}}$ de 12 , ocorreu uma leve deterioração nas características de sedimentabilidade do lodo biológico (IVL de $129 \mathrm{~mL} \cdot \mathrm{g}^{-1}$ de lodo biológico), porém houve menor perda de massa em relação aos ensaios anteriores (7\%).

\section{CONCLUSÕES}

No tratamento de lixiviado proveniente de aterro sanitário antigo, a redução das formas nitrogenadas $\left(\mathrm{N}-\mathrm{NO}_{2}\right.$ e $\left.\mathrm{N}-\mathrm{NO}_{3}\right)$ não é eficiente devido à baixa razão $\mathrm{C} / \mathrm{N}$ do efluente bruto. Além disso, o sistema sofre a oxidação anaeróbia do nitrogênio amoniacal proveniente do efluente bruto (Annamox), provocando aumento de concentração de nitrato e nitrito no reator.

O melaço de cana como fonte de carbono aumentou consideravelmente a taxa específica de desnitritação via nitrito, auxiliando na remoção de nitrogênio do efluente. As taxas específicas de desnitritação foram crescentes $\left(0,07 ; 0,19\right.$ e 0,12 kg nitrito. $\mathrm{kg}^{-1}$ $\mathrm{SSV}^{\star}$ dia) para a relação $\mathrm{DBO} / \mathrm{N}-\mathrm{NO}_{\mathrm{x}}$ crescente (de 0,$37 ; 4$ e 14, respectivamente). A ampliação do TDH também propiciou o aumento da desnitritação, com aumento da taxa específica de desnitritação. Operando-se o reator desnitrificante sob razão $\mathrm{DBO} / \mathrm{N}-\mathrm{NO}_{\mathrm{x}} \mathrm{de}$ 12 e com tempo de fase anóxica total de $22 \mathrm{~h}$, a taxa específica de desnitritação foi de $0,55 \mathrm{~kg}$ nitrito. $\mathrm{kg}^{-1} \mathrm{SSV}^{\star}$ dia.

Apesar de o melaço de cana ter proporcionado aumento significativo na eficiência da desnitrificação, não foi capaz de remover totalmente o nitrato e nitrito presentes no reator. Outras fontes externas de carbono devem ser avaliadas como forma de otimizar a desnitrificação via nitrito e via nitrato e comparar custos versus benefícios.

\section{AGRADECIMENTOS}

Os autores gostariam de agradecer à Coordenação de Aperfeiçoamento de Pessoal de Nível Superior (CAPES) pelo suporte financeiro, à Proactiva Meio Ambiente e à Fundação Universidade Regional de Blumenau (FURB) pelo suporte técnico prestado. 


\section{REFERÊNCIAS}

AHN, Y.H. (2006) Sustainable nitrogen elimination biotechnologies: a review. Process Biochemistry, v. 41, p. 1709-1721.

AMARAL, M.C.S. (2008) Avaliação da biodegradabilidade anaeróbia de lixiviados de aterros sanitários. Engenharia Sanitária e Ambiental, v. 13, n. I, p. 38-45.

APHA. (2005) Standard Methods for the Examination of Water and Wastewater. Baltimore: Port City Press.

AUN, M.V. (2007) Estudo da remoção de nitrogênio via nitrito e via nitrato em sistemas de lodo ativado alimentados pordespejo com elevada concentração de fenol. Tese (Doutorado em Engenharia), Universidade de São Paulo, São Paulo.

BUTKOVSKYI, A. (2009) Leachate Treatment at Filborna Landfill with Focus on Nitrogen Removal. Master`s Thesis, Department of Chemical Engineering, Lund University, Sweden.

CAMPOS, J.L.; GARRIDO, J.M.; MOSQUERA-CORRAL, A.; MÉNDEZ, R. (2007) Stability of a nitrifying activated sludge reactor. Biochemical Engineering Journal, v. 35, p. 87-92.

CARRERA, J.; VICENT, T.; LAFUENTE, F.J. (2003) Influence of temperature on denitrification of an industrial high-strength nitrogen wastewater in a two-sludge system. Water SA, v. 29, n. 1.

ESTUARDO, C:; MARTI, M.C:; HUILIÑIR, C:; ASPÉ, E.; ROECKEL, M. (2003) Improvement of nitrate and nitrite reduction rates prediction. Electronic Journal of Biotechnology, v. 11, n. 3, p. 1-10.

FREITAS, B.O. (2009) Remoção de nitrogênio de lixiviado de residuos sólidos urbanos por meio do processo nitrificação/desnitrificação via nitrito em reator em bateladas seqüenciais. Dissertação (Mestrado em Tecnologia Ambiental e Recursos Hídricos) - Universidade de Brasília, Brasília.

GOMES, L.P. (2009) Estudos de caracterização e tratabilidade de lixiviados de aterros sanitários para as condições brasileiras. Rio de Janeiro: ABES.

GOPAL, A.R. \& KAMMEN, D.M. (2009) Molasses for ethanol: the economic and environmental impacts of a new pathway for the lifecycle greenhouse gas analysis of sugarcane ethanol. IOP Publishing, Environmental Research Letters, v. 4.

LANGE, L. C.; ALVES, J. F.; AMARAL, M. C. S.; MELO JUNIOR, W. R. (2006) Tratamento de lixiviado de aterro sanitário por processo oxidativo avançado empregando reagente de Fenton. Engenharia Sanitária e Ambiental, v. II, n. 2, p. 175-183.
MARINGONDA JUNIOR, A. (2008) Remoção de Nitrogênio de lixiviado de aterros de resíduos sólidos urbanos empregando sistema de lodos ativados, composto por reator anóxico e aeróbio em série. Dissertação (Mestrado em Engenharia de Edificações e Saneamento Universidade Estadual de Londrina, Londrina).

PIACENTE, F.J. (2005) Agroindústria canavieira e o sistema de gestão ambiental: o caso das usinas localizadas nas bacias hidrográficas dos rios Piracicaba, Capivari e Jundiaí. Dissertação (Mestrado em Desenvolvimento Econômico) - Universidade Estadual de Campinas, Campinas.

QUEIROZ, L.M.; AUN, M.V; MORITA, D.M.; ALEM SOBRINHO, P.(2O11) Biological Nitrogen Removal over Nitritation/Denitritation using phenol as carbon source. Brazilian Journal of Chemical Engineering, v. 28, n. 2, p. 197-207.

RODRIGUES, M.R.M. (2007) Tratabilidade do lixiviado efluente da lagoa facultativa do aterro de Curitiba por lodos ativados. Dissertação (Mestrado) em Engenharia de Recursos Hídricos e Ambiental Universidade Federal do Paraná, Curitiba.

SILVA, A.C. (2002) Tratamento do percolado de aterro sanitário e avaliação da toxicidade do efluente bruto e tratado. Dissertação (Mestrado em Engenharia Civil) - Universidade Federal do Rio de Janeiro, Rio de Janeiro.

SPAGNI, A.; MARSILI-LIBELLI, S.; LAVAGNOLO, M.C. (2008) Optimisation of sanitary landfill leachate treatment in a sequencing batch reactor. Water Science \& Techonology, v. 58, n. 2, p. 337-343.

TENGRUI, L.; AL-HARBAWI, A.F.; BO, L.M.; JUN, Z.; LONG, X.Y. (2007) Characteristics of Nitrogen Removal from Old Landfill Leachate by Sequencing Batch Biofilm Reactor. American Journal of Applied Sciences, v. 4, n. 4, p. 211-214.

VAN HAANDEL A.; KATO, M.; VON SPERLING, M. (2009) Remoção Biológica de Nitrogênio: Aplicação para o Sistema de Lodo Ativado. In: MOTA, F.S.B.; VON SPERLING, M. (Coord.). Nutrientes de esgoto sanitário: utilização e remoção. Rio de Janeiro: ABES.

VON SPERLING, M. (1997) Princípios do tratamento biológico de águas residuárias - lodos ativados. v. 4. Belo Horizonte: Departamento de Engenharia Sanitária e Ambiental; Universidade Federal de Minas Gerais.

VON SPERLING, M.; ANDRADE NETO, C.O.; VOLSCHAN JÚNIOR, I.; FLORÊNCIO, L. (2009) Impacto dos Nutrientes do Esgoto Lançado em Corpos de Água. In: MOTA, F.S.B.; VON SPERLING, M. (Coord.). Nutrientes de esgoto sanitário: utilização e remoção. Rio de Janeiro: ABES.

ZAFARZADEH, A., BINA, B., NIKAEEN, M., ATTAR, H.M., KHIADANI, M.H. (2011) Effect of dissolved oxygen and chemical oxygen demand to nitrogen ratios on the partial nitrification/denitrification process in moving bed biofilm reactors. Iranian Journal of Biotechnology, v. 9, n. 3, p. 197-205. 